\title{
Analysis of mode-locked internally frequency doubled broad-band continuous wave lasers
}

\author{
H K T KUMAR, K APPAJI GOWDA* and K P J REDDY ${ }^{+}$ \\ Department of Physics, Siddaganga Institute of Technology, Tumkur 572 103, India \\ *Department of Physics, Bangalore University, Jnana Bharathi Campus, Bangalore 560056, India \\ + Department of Aerospace Engineering, Indian Institute of Science, Bangalore 560012, India
}

MS received 17 August 1994; revised 25 November 1994

\begin{abstract}
Closed-form analysis of pulse evolution in the transient regime in an actively modelocked internally frequency doubled broad-band continuous wave laser is presented. The analysis enables investigation of the effect of nonlinear crystal inside the laser cavity on mode-locked pulse parameters at the fundamental frequency. It is shown that the presence of internal second harmonic crystal broadens the fundamental mode-locked pulse while accelerating the approach of the system to steady-state. The dependence of pulse parameters on the bandwidth of the tuning element, modulation depth of the active mode-locker and conversion efficiency of the frequency doubler is presented in detailed graphical form.
\end{abstract}

Keywords. Broad-band lasers; mode locking; Picosecond pulses; frequency-doubling; pulsewidth-band-width product.

\section{PACS Nos $42 \cdot 55 ; 42 \cdot 60 ; 42 \cdot 65$}

\section{Introduction}

The wide range of applications of wavelength tunable ultrashort light pulses in scientific, medical and industrial research has led to the concentrated efforts towards the development of reliable ultrashort pulse laser sources. Stringent requirements on the stability of laser pulses for these applications have eliminated the usage of conventionally recognised dye laser sources which suffer from photodegradation of the dye solutions. As a consequences tunable broad-band solid state lasers have been recently evolved as alternate sources in the near-infrared and visible region. Among these lasers were the broad-band $\mathrm{Ti}: \mathrm{Al}_{2} \mathrm{O}_{3}$ laser with tunability ranging from 670 to $1100 \mathrm{~nm}$ pulses has clearly evolved as an excellent source of ultrashort light pulses for many applications including high resolution spectroscopy [1].

Mode-locking is the chief means of generating ultrashort light pulses from lasers [2]. All the predominant techniques, such as, active [3-5], passive [6], self [7], synchronous [8], additive-pulse [9,10], resonant passive [11] and coupled-cavity [12] mode- locking, have been employed to generate ultrashort pulses from the broad-band solid state lasers. The shortest pulses of $11 \mathrm{fs}$ duration have been generated in a self-mode-locked Ti: sapphire laser without the help of any additional pulse-compression techniques [13-15].

The operating range of the mode-locked Ti:sapphire laser has been extended into the blue region of $335-550 \mathrm{~nm}$ by either intracavity frequency doubling or second harmonic generation outside the laser cavity [16-19].

Although the shortest pulses are obtained from self-mode-locking of broad-band lasers, active mode-locking still holds promise as an important method to generate 


\section{H K T Kumar et al}

short pulses with greater stability and tunability from broad-band solid state lasers. The first detailed study of the characteristics of active mode-locking of lasers with broad-band dispersive media, including the effect of band width reducing elements was reported recently [20] based on the theory of active mode-locking developed in [21]. However, the effect of inclusion of SHG crystal inside the broad-band solid state laser cavity is not included in this study, although it is well known that the nonlinear crystal broadens the width of the mode-locked pulse at fundamental wavelength [22-26]. A brief report of the effect of the SHG crystal on the pulsewidth in a mode-locked internally frequency doubled (MLFD) Ti:sapphire laser at steadystate has been reported recently by Reddy [27].

The pulse inside a mode-locked laser builds up from the initial continuous wave oscillations after a lapse of definite time [28-31]. For the design of a laser system it is essential to determine the time interval required to complete the mode-locking process or the time interval required to recover if mode-locked laser is perturbed. The aim of this paper is to present the theoretical analysis of pulse evolution inside an actively mode-locked internally frequency doubled broad-band solid state laser with a special emphasis on Ti:sapphire laser. The pulse evolution dynamics in this system differs from the simple AM mode-locked system studied earlier [28] due to the inclusion of pulse broadening effect by the nonlinear crystal inside the laser cavity. In the case of AM mode-locked system the steady-state is attained when the pulse compression effect of AM modulator is balanced by the pulse broadening due to the bandwidth limiting effect of the laser medium. Whereas in the present system the steady-state is achieved when the pulse compression effect of the modulator is balanced by the combined broadening effects of the laser medium and the nonlinear doubling crystal inside the laser cavity. This may affect the time required for the mode-locked pulse to reach steady-state. The present analysis provides means to compute exact number of round-trips the pulse makes inside the laser cavity to reach steady-state. In addition, the analysis results in an analytic expression for the mode-locked pulsewidth and pulsewidth-bandwidth products in terms of the system parameters, such as, gain bandwidth, tuning element bandwidth and the SHG conversion efficiency of the nonlinear crystal.

\section{Analysis}

In essence the mode-locked pulse developes from the large number of small pulses present in the initial noise-like emission in the laser cavity. Of many such pulses only one pulse with repetition time defined by the cavity round-trip time survives and undergoes the pulse broadening and compression effects of the elements in each round-trip inside the cavity, ultimately reaching a steady-state. This process of pulse evolution can be analysed by using the self-consistent approach developed by Kuizenga and Siegman [21] for the time-domain description of active mode-locking in solid state lasers. A schematic description of this model is presented in figure 1. As a starting point we assume the existence of an optical pulse inside the laser cavity with an electric field described in the Gaussian form as

$$
E(t)=\left(E_{o} / 2\right) \exp \left(-\gamma t^{2}+j \omega_{p} t\right)
$$

where $\gamma=\alpha-\mathrm{j} \beta$ is the complex shape factor, $\omega_{p}$ is the centre frequency of the optical pulse and $\alpha$ and $\beta$ are related to the pulsewidth $\tau_{p}$ and pulsewidth-bandwidth 
product $\tau_{p} \Delta f_{p}$ as

and

$$
\tau_{p}=[(2 \ln 2) / \alpha]^{1 / 2}
$$

$$
\tau_{p} \Delta f_{p}=(2 \ln 2) / \pi\left[1+(\beta / \alpha)^{2}\right]^{1 / 2}
$$

Thus the parameter $\alpha$ determines the width of the Gaussian pulse in time-domain while the parameter $\beta$ indicates whether the pulse is chirped or not.

Following the schematic in figure 1, we first consider the passage of the pulse through the amplifier medium. Assuming that the gain medium is homogeneously broadened, the amplitude gain is considered in the Lorentzian form. For operation near the gain peak we can approximate the Lorentzian gain shape with a Gaussian in the form

$$
g(\omega)=\exp \left[g-4 g\left(\omega-\omega_{a}\right)^{2} / \Delta \omega_{a}^{2}\right]
$$

near the line centre (after dropping the phase term). Here $g$ is the saturated round- trip amplitude gain.

Thus, the Gaussian pulse remains Gaussian upon passage through the laser medium with the modified shape parameter

$$
\frac{1}{\gamma^{\prime}}=\frac{1}{\gamma}+\frac{16 g}{\Delta \omega_{a}^{2}}
$$

Hence if the fractional change in $\gamma$ is small, the net change due to the passage through the amplifier is

$$
\Delta \gamma_{A}=-\frac{16 g \gamma^{2}}{\Delta \omega_{a}^{2}}
$$

This indicates the broadening of the pulse due to bandwidth narrowing effect of the amplifier.

Next the effect of SHG crystal on the pulse is considered in which it is assumed that the effect of many passes through the crystal is to alter only Gaussian shape parameter and amplitude, while essentially maintaining the Gaussian shape [22]. Thus, the pulse emerging from the crystal after one round-trip will have the field amplitude in the form

$$
E^{\prime}(t)=\left(\frac{E_{o}}{2}\right) e^{a+\alpha_{0}} \exp \left(-\gamma b t^{2}\right)
$$

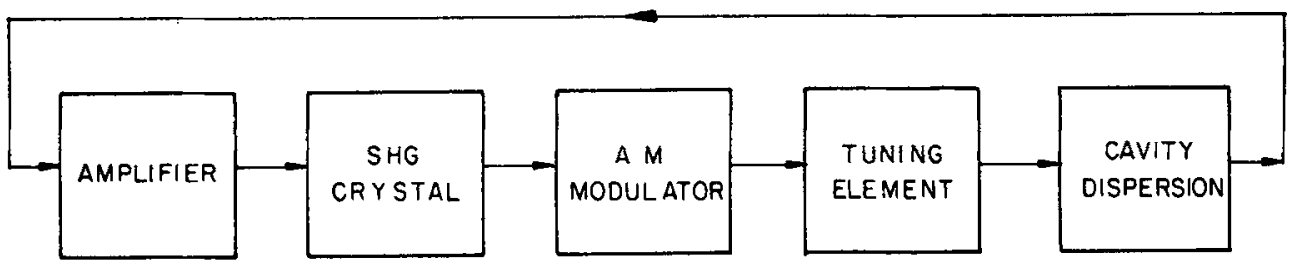

Figure 1. Block diagram of the round-trip model for pulse buildup inside an MLFD laser cavity. 


\section{H K T Kumar et al}

where $a=\sqrt{2} \alpha_{s}, b=1-2 \sqrt{2} \alpha_{s}, \alpha_{s}$ is the average single pass SHG conversion efficiency and $\alpha_{o}$ is the linear insertion loss of the crystal. Thus the net change in the pulse shape parameter in one round-trip through the doubling crystal is

$$
\Delta \gamma_{s}=-2 \sqrt{2} \alpha_{s}
$$

The negative sign in equations (6) and (8) indicate that the passage through the amplifier and the doubling crystal has reduced the magnitude of the shape parameter, which implies broadening of the pulse.

Active mode-locking could be achieved either by an AM or FM modulator inside the laser cavity. The transmission of these modulators can generally by expressed as $\exp \left[-\delta_{g}\left(\omega_{m} t\right)^{2}\right]$ where $\delta_{g}=\mp j \delta_{c}$ for $\mathrm{FM}$ modulator and $\delta_{g}=2 \delta_{l}$ for the AM modulator, $\delta_{c}$ is the effective single-pass phase retardation of the FM modulator and $\delta_{l}$ is the modulation index of AM modulator [21]. Since both AM and FM active mode-locking process are similar, we consider only AM mode-locking in the present analysis. The pulse shape parameter undergoes a change $\Delta \gamma_{\mathrm{AM}}$ due to a round-trip passage through the AM modulator. This is given by

$$
\Delta \gamma_{\mathrm{AM}}=2 \delta_{l} \omega_{m}^{2}
$$

The above equation indicates that the passage of the mode-locked pulse through the AM modulator undergoes an enhancement in the shape parameter leading to the pulse compression.

It is important to note that when the pulse passes through the host media of the amplifier, mode-locker and the frequency doubling crystal, it suffers change due to dispersion effects. These effects are taken into account in the present analysis by considering the transmission function for the bandwidth limiting element such as multiple plate birefringent filter, in the following form [20]

$$
\begin{aligned}
& T(\omega)=\exp \left[-j\left(\left.P\right|_{\lambda=\lambda_{a}} \omega / c\right)\right] \exp \left[(j / c)\left\{\lambda_{a}^{2}\left[\mathrm{~d} /\left.\mathrm{d} \lambda(P / \lambda)\right|_{\lambda=\lambda_{a}}\right]\left(\omega-\omega_{a}\right)\right\}\right] \\
& \times \exp \left[\left(-4 / \Delta \omega_{t}^{2}-j D / 4 \pi^{2}\right)\left(\omega-\omega_{a}\right)^{2}\right]
\end{aligned}
$$

where $P$ is the round-trip optical path length, $c$ is the speed of light in vacuum, $\lambda_{a}$ is the wavelength of light at the gain peak, $\Delta \omega_{t}$ is the bandwidth of the tuning element, and $D=\left[\frac{\lambda_{a}^{3}}{8 c^{2}}\right]\left[\left.\frac{d^{2} P}{d \lambda^{2}}\right|_{\lambda=\lambda_{a}}\right]$ represents the round-trip cavity group velocity dispersion.

The operation of this transfer function essentially maintains the Gaussian shape of the mode-locked pulse, while inducing a change in the shape parameter given by

$$
\Delta \gamma_{\text {cavity }}=-\left(\frac{16 \gamma^{2}}{\Delta \omega_{t}^{2}}+j \frac{4 D \gamma}{\pi^{2}}\right)
$$

Thus it can be seen that the bandwidth limiting element enhances the width of the mode-locked pulse while the group velocity dispersion essentially contributes to the chirp of the pulse.

Now we are in a position to estimate the net rate of change in the shape parameter due to a round-trip travel through the cavity elements in an MLFD broad-band laser. 


\section{Mode-locked frequency doubled laser}

This is achieved by adding Equations (6), (8), (9) and (11), which yields

$$
\left(\frac{\Delta \gamma}{\Delta t}\right)=\left[2 \delta_{l} \omega_{m}^{2}-2 \sqrt{2} \alpha_{s} \gamma-16 \gamma^{2}\left\{\frac{g}{\Delta \omega_{a}^{2}}+\frac{1}{\Delta \omega_{t}^{2}}+j \frac{D}{4 \pi^{2}}\right\}\right]\left(\frac{c}{2 L}\right)
$$

where $\Delta t$ is the cavity round-trip time interval, and $L$ is the length of the laser resonator.

Assuming the net rate of change of the shape parameter in a single cavity round trip is small, the above equation can be written in the differential form

$$
\frac{\mathrm{d} \gamma}{\mathrm{d} t}=\left\{2 \delta_{l} \omega_{m}^{2}-2 \sqrt{2} \alpha_{s}-16\left(\frac{g}{\Delta \omega_{a}^{2}}+\frac{1}{\Delta \omega_{t}^{2}}+j \frac{D}{4 \pi} \gamma^{2}\right)\right\}\left(\frac{c}{2 L}\right)
$$

where we have replaced $\mathrm{d} \gamma / \mathrm{d} t \equiv \Delta \gamma / \Delta t, \Delta t \approx 2 L / c$. The slight change in the round-trip time interval due to dispersion effect of the materials inside the laser resonator is neglected.

\section{Results and discussion}

The evolution of the mode-locked pulse in the transient regime is defined by the first order differential equation (12). However, before solving the equation for transient solution, an estimation of the steady-state pulsewidth can be obtained by assuming very large time parameter. Thus the mode-locking equation yields the solution for $\gamma$ at steady-state in the form

$$
\begin{aligned}
\gamma= & {\left[16\left(\frac{g}{\Delta \omega^{2}}+\frac{1}{\Delta \omega_{t}^{2}}+j \frac{D}{4 \pi^{2}}\right)\right]^{-1} } \\
& \left\{-\sqrt{2} \alpha_{s}+\left[\left(\sqrt{2} \alpha_{s}\right)^{2}+32 \delta_{l} \omega_{m}^{2}\left(\frac{g}{\Delta \omega^{2}}+\frac{1}{\Delta \omega_{t}^{2}}+j \frac{D}{4 \pi^{2}}\right)\right]^{1 / 2}\right\}
\end{aligned}
$$

where we have assumed $\alpha_{s} \ll 1$, which is essential to avoid overcoupling of the laser power, and also the pulse spectral width is assumed to be much smaller than the laser linewidth.

This equation is identical to the corresponding equation of Reddy [27] which yields for higher values of SHG conversion efficiency the mode-locked pulsewidth

$$
\tau_{p}=\frac{\left(\sqrt{2} \ln 2 \alpha_{s}\right)^{1 / 2}}{\pi f_{m}\left(2 \delta_{l}\right)^{1 / 2}}
$$

Hence in the high SHG regime, the mode-locked pulsewidth is independent of the laser gain and the bandwidths of the laser medium and the tuning element while it increases directly with the increase of SHG conversion efficiency.

The steady-state pulsewidth and pulsewidth-bandwidth product are obtained for moderate values of SHG conversion efficiency by separating the real and imaginary parts $\alpha$ and $\beta$ from (13) which yields

$$
\tau_{p s}=(2 \ln 2)^{1 / 2} A^{1 / 2}\left\{\left(\frac{g}{\Delta \omega_{a}^{2}}+\frac{1}{\Delta \omega_{t}^{2}}\right)\left[(r+R)^{1 / 2}-2 \alpha_{s}\right]-\left(\frac{D}{4 \pi^{2}}\right)(r-R)^{1 / 2}\right\}^{1 / 2}
$$


and

$$
\begin{aligned}
\tau_{p s} \Delta f_{p s} & =(2 \ln 2 / \pi) \\
& \left\{1+\left[\frac{\left(D / 4 \pi^{2}\right)\left[(r+R)^{1 / 2}-2 \alpha_{s}\right]-\left(g / \Delta \omega^{2}+1 / \Delta \omega_{t}^{2}\right)(r-R)^{1 / 2}}{\left(g / \Delta \omega^{2}+1 / \Delta \omega_{t}^{2}\right)\left[(r+R)^{1 / 2}-2 \alpha_{s}\right]-\left(D / 4 \pi^{2}\right)(r-R)^{1 / 2}}\right]^{2}\right\}^{1 / 2}
\end{aligned}
$$

where

and

$$
\begin{aligned}
r & =\left(R^{2}+I^{2}\right)^{1 / 2} \\
R & =2 \alpha_{s}^{2}+32 \delta_{l} \omega_{m}^{2}\left(g / \Delta \omega^{2}+1 / \Delta \omega_{t}^{2}\right) \\
I & =32 \delta_{l} \omega_{m}^{2}\left(D / 4 \pi^{2}\right)
\end{aligned}
$$

$$
A=16 \sqrt{2}\left[\left(g / \Delta \omega^{2}+1 / \Delta \omega_{t}^{2}\right)^{2}+\left(D / 4 \pi^{2}\right)^{2}\right] .
$$

These equations yield the mode-locked pulsewidth and pulsewidth-bandwidth product as functions of the system parameters in an MLFD broad-band laser system at steady-state. These equations can be used to analyse the dependence of pulse parameters on the SHG conversion efficiency, modulation depth and the tuning element bandwidth. However, since the main aim of the present paper is to study the transient evolution of the pulse parameters, we concentrate further discussions on the transient solutions of the mode-locking equation (12), which are in the form

where

$$
\gamma=\gamma_{0} \tanh \left[(\sigma+j \Omega)\left(t+t_{0}\right)(c / 2 L)\right]-K
$$

and

$$
\begin{aligned}
\gamma_{o}= & {\left[8 \alpha_{s}^{2}+64 \delta_{l} \omega_{m}^{2}\left(g / \Delta \omega_{a}^{2}+1 / \Delta \omega_{t}^{2}+j D / 4 \pi^{2}\right)\right]^{1 / 2} } \\
& {\left[32\left(g / \Delta \omega_{a}^{2}+1 / \Delta \omega_{t}^{2}+j D / 4 \pi^{2}\right)\right]^{-1} } \\
\sigma= & \operatorname{Re}\left[8 \alpha_{s}^{2}+64 \delta_{l} \omega_{m}^{2}\left(g / \Delta \omega_{a}^{2}+1 / \Delta \omega_{t}^{2}+j D / 4 \pi^{2}\right)\right]^{1 / 2} \\
\Omega= & \operatorname{Im}\left[8 \alpha_{s}^{2}+64 \delta_{l} \omega_{m}^{2}\left(g / \Delta \omega_{a}^{2}+1 / \Delta \omega_{t}^{2}+j D / 4 \pi^{2}\right)\right]^{1 / 2}
\end{aligned}
$$

$$
K=2 \sqrt{2} \alpha_{s}\left[16\left(g / \Delta \omega_{a}^{2}+1 / \Delta \omega_{t}^{2}+j D / 4 \pi^{2}\right)\right]^{1 / 2} .
$$

Separating real and imaginary parts, equation (17) yields

$$
\begin{aligned}
\alpha= & \left\{\left[\left(g / \Delta \omega_{a}^{2}+1 / \Delta \omega_{t}^{2}\right)(r+R)^{1 / 2}+\left(D / 4 \pi^{2}\right)(r-R)^{1 / 2}\right] \sinh (2 \sigma M)\right. \\
& \left.-\left[\left(g / \Delta \omega_{a}^{2}+1 / \Delta \omega_{t}^{2}\right)(r-R)^{1 / 2}-\left(D / 4 \pi^{2}\right)^{2}(r+R)^{1 / 2}\right] \sinh (2 \Omega M)\right\} \\
& {[(\cosh (2 \sigma M)+\cos (2 \Omega M)) A]^{-1}-2 \alpha_{s}\left(g / \Delta \omega_{a}^{2}+1 / \Delta \omega_{t}^{2}\right) A^{-1} } \\
\beta= & \left\{\left[\left(g / \Delta \omega_{a}^{2}+1 / \Delta \omega_{t}^{2}\right)(r+R)^{1 / 2}+\left(D / 4 \pi^{2}\right)(r-R)^{1 / 2}\right] \sin (2 \Omega M)\right. \\
& \left.-\left[\left(g / \Delta \omega_{a}^{2}+1 / \Delta \omega_{t}^{2}\right)(r-R)^{1 / 2}-\left(D / 4 \pi^{2}\right)(r+R)^{1 / 2}\right] \sinh (2 \sigma M)\right\} \\
& {[(\cosh (2 \sigma M)+\cos (2 \Omega M)) A]^{-1}+2 \alpha_{s} D / 4 \pi^{2} }
\end{aligned}
$$

where $M=(c / 2 L)\left(t+t_{o}\right)$ is the number of round-trips.

The above equations define the pulsewidth and pulsewidth-bandwidth products of the mode-locked pulse inside an MLFD broad-band laser as a function of number of round-trips inside the laser cavity. For large number of round-trips these equations reduce to the form given in (15) and (16) for steady-state. Also, in absence of SHG the transient regime equations reduce to the form given by Reddy and Tatum [28] for 


\section{Mode-locked frequency doubled laser}

simple active mode-locking case. At steady-state if we assume the case of active mode-locking without frequency doubling, these equations reduce to the form given by Darrow and Jain [20] under the approximations, $g / \Delta \omega_{a}^{2}<1 / \Delta \omega_{t}^{2}$ and/or $D / 4 \pi^{2}$. Thus the analytical equations (18) and (19) yield the transient solutions for pulsewidth and pulsewidth-bandwidth products which are the most general form of equations to describe the simultaneous mode-locking and internal frequency doubling in broadband laser systems.

For the demonstration of the usefulness of the present theory we apply (18) and (19) to study the pulse evolution in an MLFD Ti:sapphire laser. The system parameters for this laser are given in table 1 . The variation of mode-locked pulsewidth as a function of number of cavity round-trips inside the laser cavity is shown in figure 2, for different values of SHG conversion efficiency, and modulation depth of 0.55 . In this case we have assumed that the bandwidth limiting element is a two plate briefringent filter. A noticeable feature of the curves in figure 2 is that the pulsewidth approaches steady-state value rather suddenly after few hundred round-trips. For

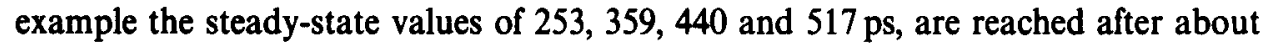
$1100,700,500$ and 400 round-trips for the SHG conversion efficiencies of $0.5,1.0,1.5$ and $2.0 \%$ respectively. This corresponds to the pulse evolution time of $3.66,2 \cdot 33,1.66$ and $1.33 \mu \mathrm{s}$ for a cavity length of $50 \mathrm{~cm}$. Essentially the $\mathrm{SHG}$ nonlinear crystal broadens the laser pulse in each transit through the crystal and this broadening effect increases with the increased conversion efficiency. This effect is seen in figure 2 , which shows that the steady-state pulsewidth increases with the increase of conversion efficiency of the doubling crystal. One more important effect observed in figure 2 is that the number of cavity round-trips at which the steady-state is approached increases with the decrease of the conversion efficiency. Similar features have been predicted for narrow band solid state lasers $[24,25]$.

The effect of bandwidth of birefringent tuning element on the pulse evolution time for different SHG conversion efficiencies is shown in figure 3. It is seen that the effect of increasing the bandwidth for a given SHG conversion efficiency is to accelerate the approach of the pulsewidth towards the steady-state. However, the effect of bandwidth variation on the steady-state pulsewidth is negligible.

The variation of the mode-locked pulsewidth with the SHG conversion efficiency is highlighted in figure 4 with modulation depth of the active mode-locking element as

Table 1. System parameters for MLFD Ti:Sapphire laser system.

\begin{tabular}{ll}
\hline Parameter & Numerical value \\
\hline$g$ & 0.18 \\
$L$ & $0.5 \mathrm{~m}$ \\
$\Delta f_{a}$ & $197.2 \mathrm{~T} \mathrm{~Hz}$ \\
$\Delta f_{t}$ & $56.2 \mathrm{~T} \mathrm{~Hz}$ (Single plate) \\
& $13.8 \mathrm{~T} \mathrm{~Hz}$ (Double plate) \\
$D$ & $3.45 \mathrm{~T} \mathrm{~Hz}$ (Three plate) \\
$f_{m}$ & {$\left[62.0+\left(18.3 \mathrm{~T} \mathrm{~Hz} / \Delta \mathrm{f}_{t}\right)\right] \times 10^{-28} \mathrm{~s}^{2}$} \\
$\delta_{l}$ & $160 \mathrm{MHz}$ \\
$\alpha_{s}$ & Variable \\
\hline
\end{tabular}


H K T Kumar et al

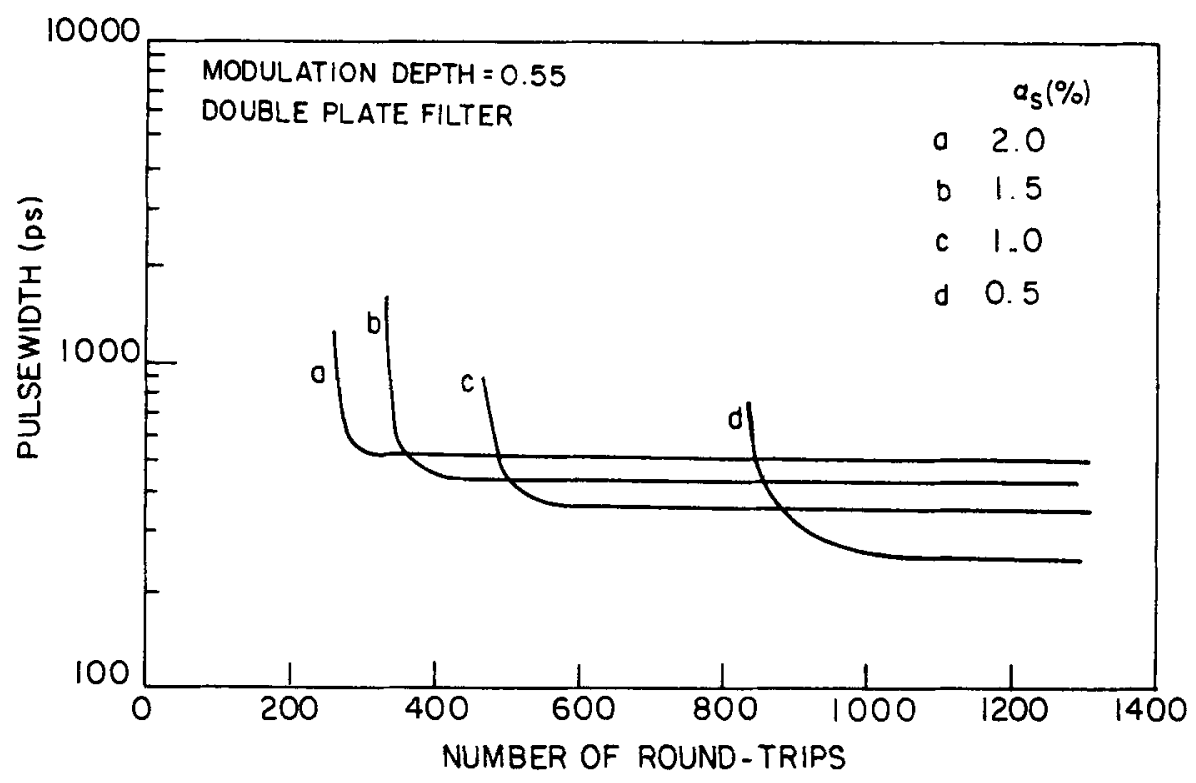

Figure 2. Dependence of the mode-locked pulsewidth on the number of roundtrips inside an MLFD laser cavity for different SHG conversion efficiencies.

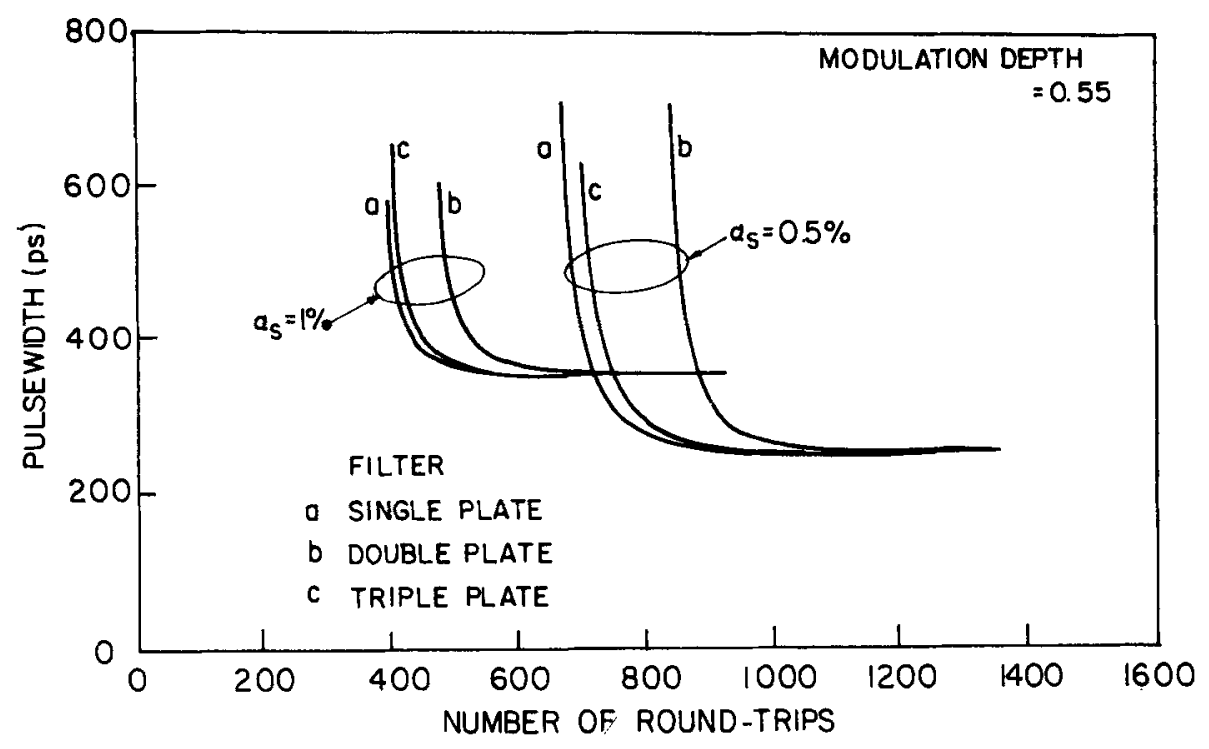

Figure 3. Effect of tuning element bandwidth on the transient evolution of mode-locked pulse inside an MLFD laser cavity.

a parameter. The starting values of all the three curves in this figure are almost identical and correspond to the pure active mode-locking values which were predicted earlier by Reddy and Tatum [28]. As pointed earlier the action of the AM modulator is to compress the pulse while that of the SHG crystal is to broaden the pulse in each transit. Hence it is seen from figure 4 that for a given modulation depth the pulsewidth 
Mode-locked frequency doubled laser

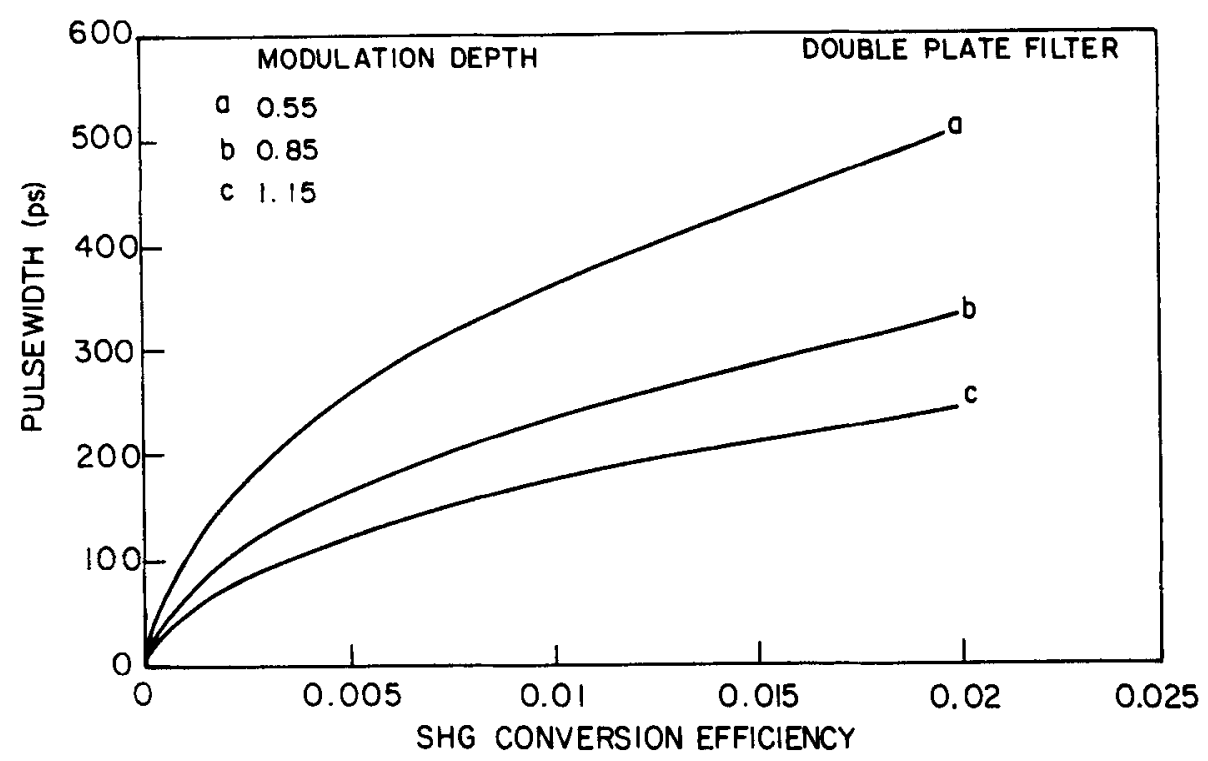

Figure 4. Dependence of the mode-locked pulsewidth at steady-state on the SHG conversion efficiency for different modulation depths of AM mode-locker.

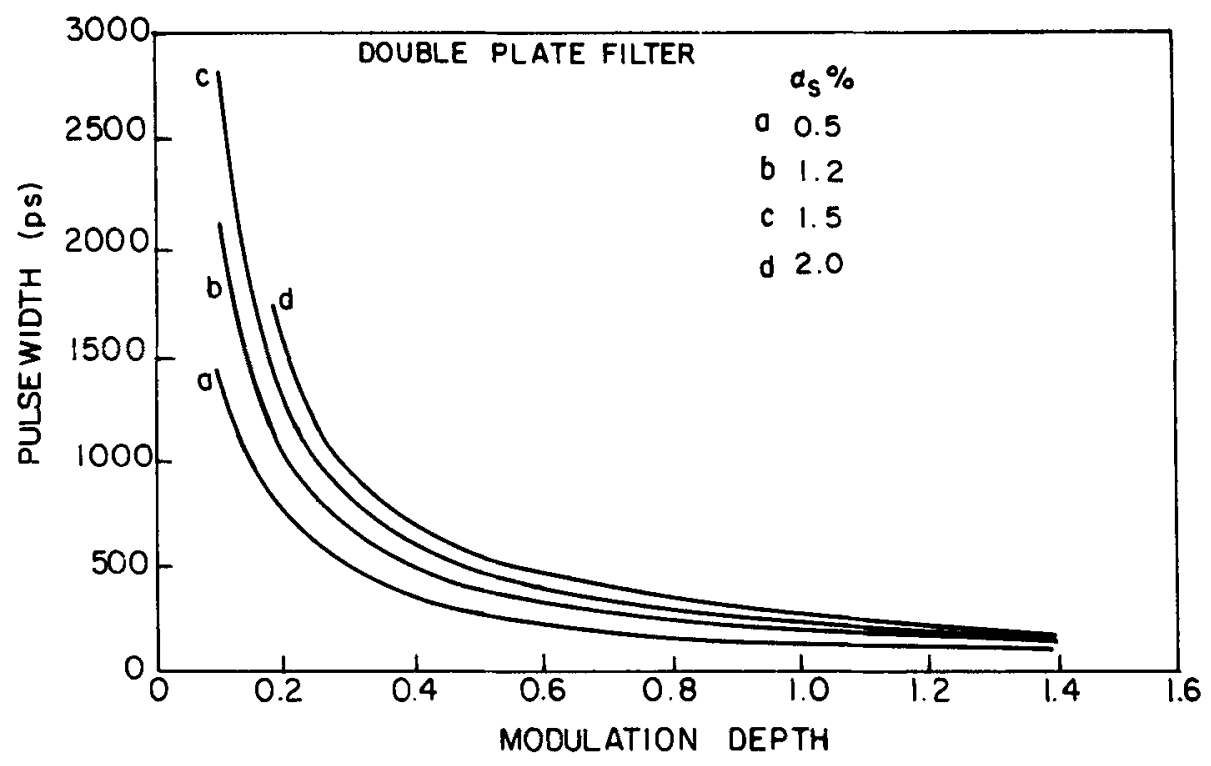

Figure 5. Dependence of the mode-locked pulsewidth at steady-state on the modulation depth of the AM mode-locker for different SHG conversion efficiencies.

increases rapidly with the increase of conversion efficiency and for a given conversion efficiency the pulsewidth decreases with the increase of modulation depth.

The dependence of steady-state pulsewidth on modulation depth of the AM modulator is shown in figure 5 where the curves are plotted for different SHG 


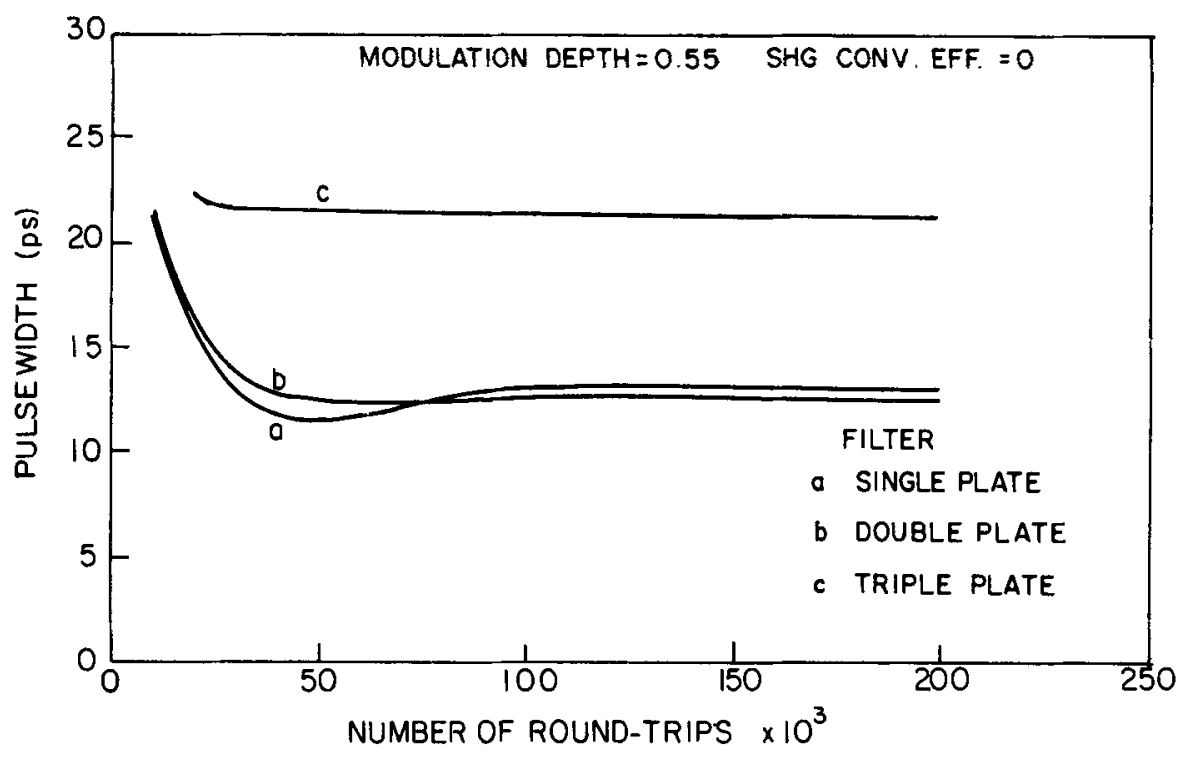

Figure 6. Transient evolution of mode-locked pulsewidth in an AM mode-locked broad-band laser for different bandwidths of the tuning element.

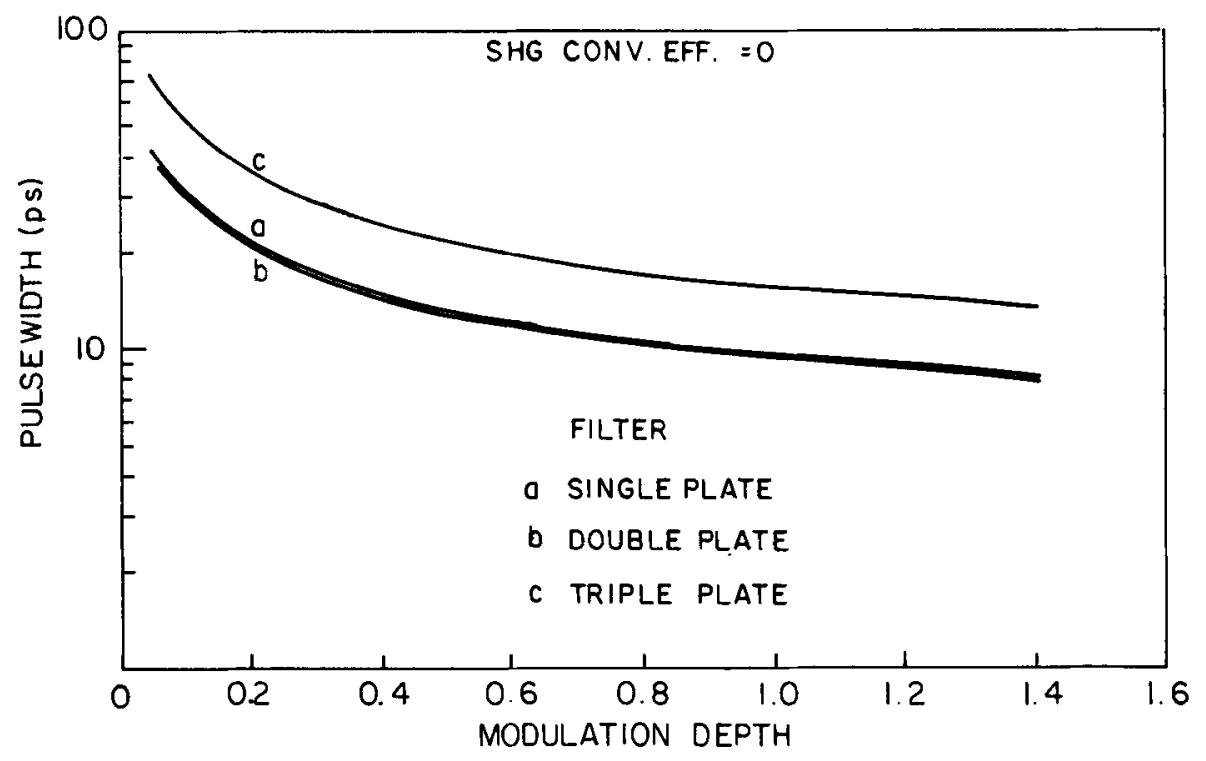

Figure 7. Dependence of steady-state mode-locked pulsewidth on the modulation depth in an AM mode-locked broad-band laser for different bandwidths of the tuning element.

conversion efficiencies. It is seen that the pulsewidth decreases with the increase of the modulation depth and the pulsewidth variation is very sensitive to the changes in the modulation depth for smaller values and becomes insensitive to changes at higher modulation depth. Hence the pulse compression ability of the AM modulator remains nearly constant beyond the modulation depth of $\sim 1$. 


\section{Mode-locked frequency doubled laser}

As a final demonstration of the general nature of the present analysis the results of Reddy and Tatum [28] are reproduced from the present analysis assuming zero SHG conversion efficiency. The results are presented in figures 6 and 7. It is seen that the number of round-trips required for the mode-locked pulsewidth to reach steady-state value predicted in the present analysis is of the same order as predicted by Reddy and Tatum [28]. Similarly, the variation of steady-state pulsewidth with the modulation depth for different tuning element bandwidths is identical to the variations shown by Reddy and Tatum [28].

\section{Conclusions}

Mode-locked broad-band solid state lasers have evolved as reliable sources of picosecond laser pulses in the visible and near infrared regions. The extension of the output wavelength of these lasers to the near ultraviolet region is achieved by frequency doubling. A comprehensive analysis of the mode-locking phenomenon in a mode-locked and internally frequency doubled broad-band laser system is presented here. The analysis enables study of the mode-locked pulse evolution in the transient and steady-state regimes. Pulse evolution studies in the transient regime has shown that the pulsewidth approaches steady-state value within few microseconds. The time taken to reach steady-state increases with the decrease of the second harmonic conversion efficiency. The analysis predicts an order of magnitude increase in the mode-locked pulsewidth due to the inclusion of intracavity second harmonic generator. The dependence of the steady-state pulsewidth on the system parameters is analysed in detail.

Although the results discussed in the present paper include only the width of the mode-locked pulse, the equations given in the paper could be utilized to study the pulsewidth-bandwidth product also. In addition, the present theory can be easily extended for the FM mode-locking in broad-band lasers.

\section{References}

[1] C S Adams and A I Ferguson, Opt. Commun. 75, 419 (1990)

[2] J D Simon, Rev. Sci. Instrum. 60, 3597 (1989)

[3] B C Johnson, P F Moulton and A Mooradian, Opt. Lett. 10, 116 (1984)

[4] C P Pinto, C P Yakymyshyn and C R Pollock, Opt. Lett. 13, 383 (1988)

[5] J T Darrow, R K Jain and M Poelker, Conf. Dig. LEOS, Wash. DC, OSA paper ME1 (1988)

[6] N Sakura, Y Ishida, H Nakano and Y Yamamoto, Appl. Phys. Lett. 56, 814 (1990)

[7] D E Spence, P N Kean and W Sibbett, Conf. Dig. LEOS, Wash. DC, OSA paper PDP10 (1990)

[8] L F Mollenauer, N D Vieira and L Szeto, Opt. Lett. 7, 414 (1982)

[9] P M W French, S M J Kelly and J R Taylor Opt. Lett. 15, 378 (1990)

[10] J Mark, L Y Liu, K L Hall, H A Haus and E P Ippen, Opt. Lett. 14, 48 (1989)

[11] V Keller, W H Knox and H Roskos, Proc. 7th Int. Conf. Ultrafast Phenom. edited by C B Harris, E P Ippen, G A Mouron and A H Zewail (Springer-Verlag, Berlin, 1990) 69

[12] L F Mollenauer and R H Stolen, Opt. Lett. 9, 13 (1984)

[13] D E Spence, P N Kean and W Sibbett, Opt. Lett. 16, 42 (1991)

[14] M T Asaki, C P Huang, D Garvey, J Zhou, H C Kapteyn and M M Murnane, Opt. Lett. 18, 977 (1993)

[15] P Curley, C Spielmann, T Brabec, F Krausz, E Wintner and A Schmidt, Opt. Lett. 18, 54 (1993) 


\section{H K T Kumar et al}

[16] C S Adams and A I Ferguson, Opt. Commun. 90, 89 (1992)

[17] V Petrov, D Georgiev and U Stamm, Appl. Phys. Lett. 60, 1550 (1992)

[18] R J Ellingson and C L Tang, Opt. Lett. 17, 343 (1992)

[19] S Backus, M T Asaki, C Shi, H C Kapteyn and M M Murnane, Opt. Lett. 19, 399 (1994)

[20] J T Darrow and R K Jain, IEEE J. Quantum Electron. 27, 1048 (1991)

[21] D J Kuizenga and A E Siegman, IEEE J. Quantum Electron. QE-6, 694 (1970)

[22] J Falk, IEEE J. Quantum Electron. QE-11, 21 (1975)

[23] M Yamashita, K Yamada and T Sato, IEEE J. Quantum Electron. QE-18, 95 (1982)

[24] K P J Reddy, J. Appl. Phys. 53, 7076 (1982)

[25] T Damm, U Stamm, K P Stolberg and B Wilhelmi, Ultrafast phenom. Spectroscopy, Springer Proc. in Phys. edited by E Klose and B Wilhelmi (Springer, Berlin, 1990) 49, 23

[26] L R Marshall, A Kaz, A D Hays and R L Burnham, Opt. Lett. 17, 1110 (1992)

[27] K P J Reddy, J. Appl. Phys. 74, 5913 (1993)

[28] K P J Reddy and J A Tatum, IEEE J. Quantum Electron. 29, 1407 (1993)

[29] J Goodberlet, J Wang and J G Fujimoto, Opt. Lett. 15, 1300 (1990)

[30] Y Ishida, N Sarukura and H Nakano, Conf. Dig. CLEO, Wash. DC, OSA paper JMB2 (1991)

[31] C L Pan, J C Kuo, K H Wu, J M Shieh, C D Hwang and C S Chang, Opt. Lett. 17, 334 (1992) 